\title{
Spontaneous Rupture of a Deep Femoral Pseudoaneurysm Mimicking Lymphedema After Radical Hysterectomy in a Woman Who Was Receiving Warfarin
}

\author{
Shun-ichi Ikeda ${ }^{\mathrm{a}, \mathrm{c}}$, Tomoko Manabe ${ }^{\mathrm{b}}$, Shunsuke Sugawara ${ }^{\mathrm{b}}$, Miyuki Sone ${ }^{\mathrm{b}}$, \\ Mitsuya Ishikawa ${ }^{\text {a }}$, Tomoyasu Kato ${ }^{\mathrm{a}}$
}

\begin{abstract}
Pseudoaneurysms of the deep femoral artery have been reported after external injury to the thigh and orthopedic surgery of the femur. We describe our experience with a case of spontaneous rupture of a deep femoral pseudoaneurysm in a woman who was receiving warfarin within the therapeutic range. She received a choledocholithotomy after a radical hysterectomy for uterine cervical cancer (stage IB1). Pulmonary embolism and deep-vein thrombosis developed after choledocholithotomy, and an inferior vena cava filter was left in place permanently. She was receiving warfarin since then. This time, the patient presented with a pseudoaneurysm of the deep femoral artery and a large hematoma in the adductor muscle of the thigh. Swelling of the thigh in this condition resembled the lower-extremity edema that occurs after pelvic lymph-node dissection. Contrast-enhanced computed tomography (CT) was useful for diagnosis of the pseudoaneurysm and evaluation of the extent of muscle damage.
\end{abstract}

Keywords: Deep femoral pseudoaneurysm; Warfarinization; Pelvic lymph node dissection; Contrast-enhanced computed tomography

\section{Introduction}

Pseudoaneurysm of the deep femoral artery has been reported occasionally after external injury to the thigh and orthopedic surgery of the femur $[1,2]$. Rarely, a spontaneous pseudoaneurysm of the deep femoral artery has developed without a clear history of trauma or orthopedic surgery of the femur under special conditions [3, 4]. Warfarin is used to treat and prevent venous thrombosis, and the dose of warfarin should be adjusted to maintain the target international normal ratio (INR)

Manuscript accepted for publication June 07, 2016

${ }^{a}$ Division of Gynecology, National Cancer Center Hospital, Tokyo, Japan bDivision of Diagnostic Radiology, National Cancer Center Hospital, Tokyo, Japan

${ }^{\mathrm{c} C o r r e s p o n d i n g ~ A u t h o r: ~ S h u n-i c h i ~ I k e d a, ~ D i v i s i o n ~ o f ~ G y n e c o l o g y, ~ N a t i o n a l ~}$ Cancer Center Hospital, 5-1-1 Tsukiji, Chuo-ku, 104-0045, Tokyo, Japan. Email: shuikeda@ncc.go.jp

doi: http://dx.doi.org/10.14740/jmc2543w
[5]. INR values that exceed the therapeutic range lead to bleeding and other complications. We report a case of spontaneous rupture of a deep femoral pseudoaneurysm that mimicked lymphedema after radical hysterectomy with pelvic lymphnode dissection in a patient who was receiving warfarin, even though the dose of warfarin was adjusted to maintain the INR within the therapeutic range.

\section{Case Report}

A 69-year-old woman noticed swelling and pain in her left thigh after doing some light stretching for about half an hour. She thought that the symptoms were caused by lymphedema developing as a complication after the radical hysterectomy as it had occurred in the past. However, she visited our hospital because she felt the pain in her thigh, which she had not experienced previously. The patient had undergone a radical hysterectomy for stage IB1 uterine cervical cancer 11 years ago. Although just after surgery, she had several episodes of lymphedema of the thigh, there was no lymphedema since then. Two years prior, she underwent a choledocholithotomy at another hospital. Immediately after surgery, she had pulmonary embolism and deep-vein thrombosis, and an inferior vena cava filter was left in place permanently. She had been receiving warfarin in a dose of $6 \mathrm{mg}$ per day since then. The patient had no history of trauma, and puncture of the femoral artery had not been performed for several years. Although the left thigh was swollen as compared with the right thigh, its skin color was normal. There was no palpable thrill in the left thigh. The hemoglobin, C-reactive protein, and INR levels were $10.3 \mathrm{~g} / \mathrm{dL}, 0.66 \mathrm{mg} / \mathrm{dL}$, and 2.19 , respectively. Subsequently, contrast-enhanced computed tomography (CT) was performed. A pseudoaneurysm of the left deep femoral artery surrounded by a large hematoma in the adductor muscle was confirmed (Fig. 1). Active bleeding from the left deep femoral pseudoaneurysm appeared to continue. Moreover, the large hematoma in the adductor muscle might progress to the compartment syndrome. Selective angiography of the left deep femoral artery was performed after the patient received $10 \mathrm{mg}$ of vitamin $\mathrm{K}$ by slow intravenous injection. The findings confirmed a 7.3-mm pseudoaneurysm in a muscular branch of the deep femoral artery (Fig. 2) and extravasation of contrast medium from the pseudoaneurysm (Fig. 3a). Coil embolization was successfully performed (Fig. 3b). Postopera- 


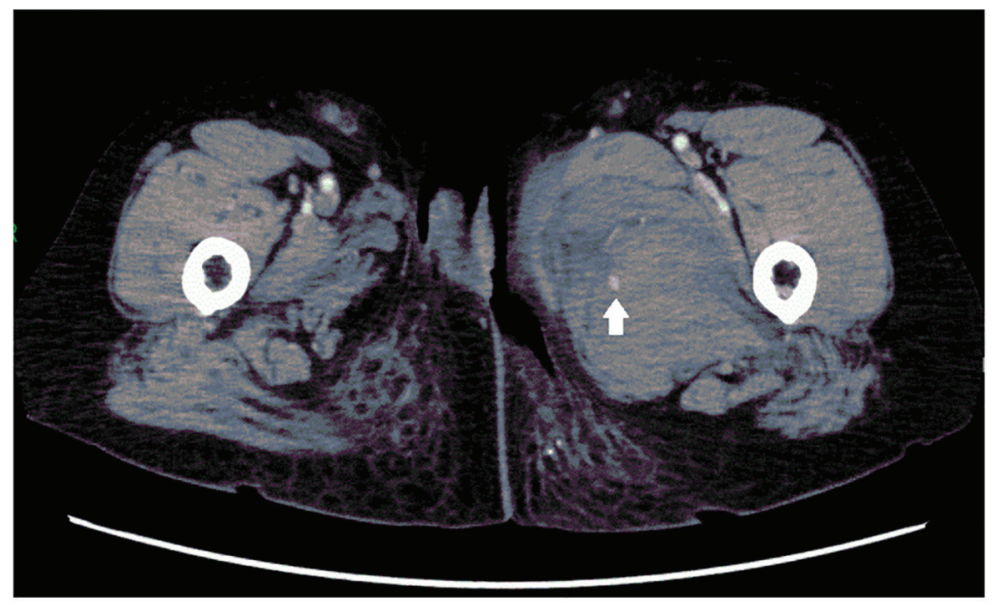

Figure 1. Contrast-enhanced CT image of the patient's left thigh, showing a pseudoaneurysm (arrow) and hematoma in the adductor compartment.

tively, the patient recovered uneventfully.

\section{Discussion}

Our experience indicated three important clinical points. First, pseudoaneurysm can develop in the deep femoral artery even when the INR level of warfarin is maintained within the therapeutic range. Second, swelling of the thigh caused by rupture of the deep femoral artery resembles the lower-extremity edema that occurs after radical hysterectomy. Third, contrastenhanced CT is useful for diagnosis of pseudoaneurysm of the deep femoral artery with hematoma and for evaluation of the

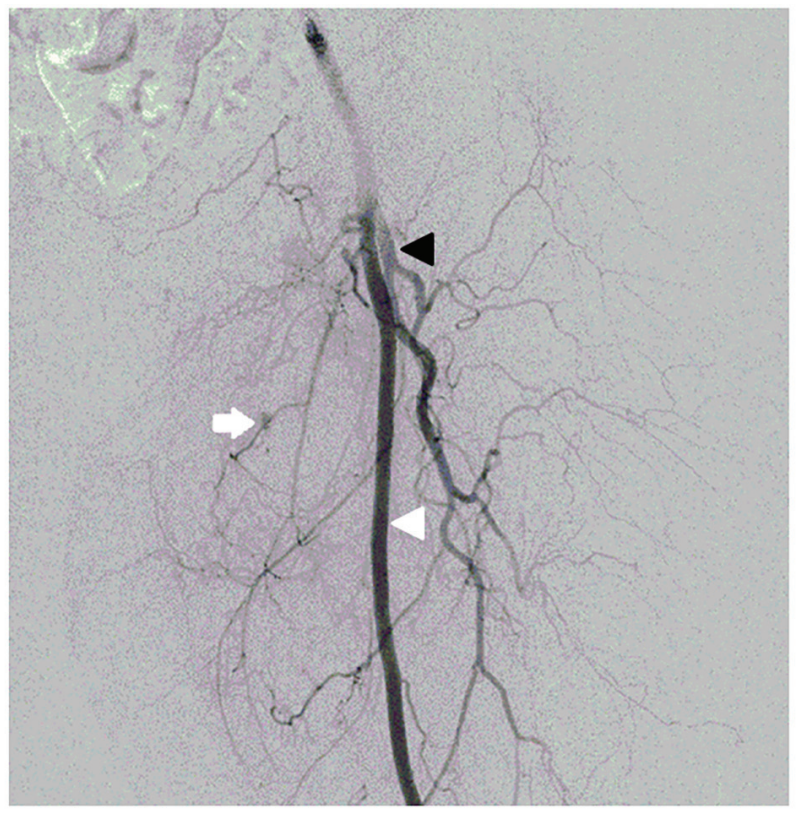

Figure 2. Angiogram showing a 7.3-mm pseudoaneurysm (arrow) in a muscular branch of the deep femoral artery. Left superficial femoral artery (white arrowhead). Left deep femoral artery (black arrowhead). extent of muscle damage caused by hematoma.

First, pseudoaneurysm can develop in the deep femoral artery even when the INR level of warfarin is maintained within the therapeutic range. Warfarin is used to treat venous thrombosis. Its safety and efficacy depend on the administered dose, and an INR value of $2.0-3.0$ is recommended [5]. A dose of warfarin exceeding the recommended therapeutic range can cause bleeding as a complication. Etiologic factors for spontaneous pseudoaneurysm formation include sports activities with muscle overcontraction or overstretching, use of anticoagulants, arterial wall weakness caused by arteriosclerosis, advanced age, inherited connective-tissue disease, infection, and congenital arterial disorders [6-8]. Our patient was an elderly (69 years old), receiving warfarin, and lightly stretched her muscles for about half an hour, before coming to our hospital. Only one of the following factors is unlikely to cause pseudoaneurysm: advanced age, warfarin treatment with an INR value of 2.19, and light stretch exercise. In our patient, the presence of all three of these factors, particularly warfarinization, probably led to the formation of a pseudoaneurysm.

Second, swelling of the thigh caused by rupture of a deep femoral pseudoaneurysm resembles the lower-extremity edema occurring after radical hysterectomy with pelvic lymph-node dissection. We often encounter patients who have lower-extremity edema after pelvic lymph-node dissection. If the patient had thought that her swollen thigh was caused by edema and massaged her thigh, bleeding might have increased considerably and caused the compartment syndrome. In patients who receive warfarin after pelvic lymph-node dissection, thigh swelling is likely to be caused not only by edema, but also potentially by femoral pseudoaneurysm. Moreover, rupture of a femoral pseudoaneurysm can directly cause thigh edema [9]. These factors might create difficulty in the diagnosis of rupture of a femoral pseudoaneurysm after pelvic lymphnode dissection.

Third, contrast-enhanced CT is useful for the diagnosis of pseudoaneurysm and for evaluation of the extent of muscle damage. Although color Doppler imaging is a sensitive and specific modality for the evaluation of pseudoaneurysm [10], 

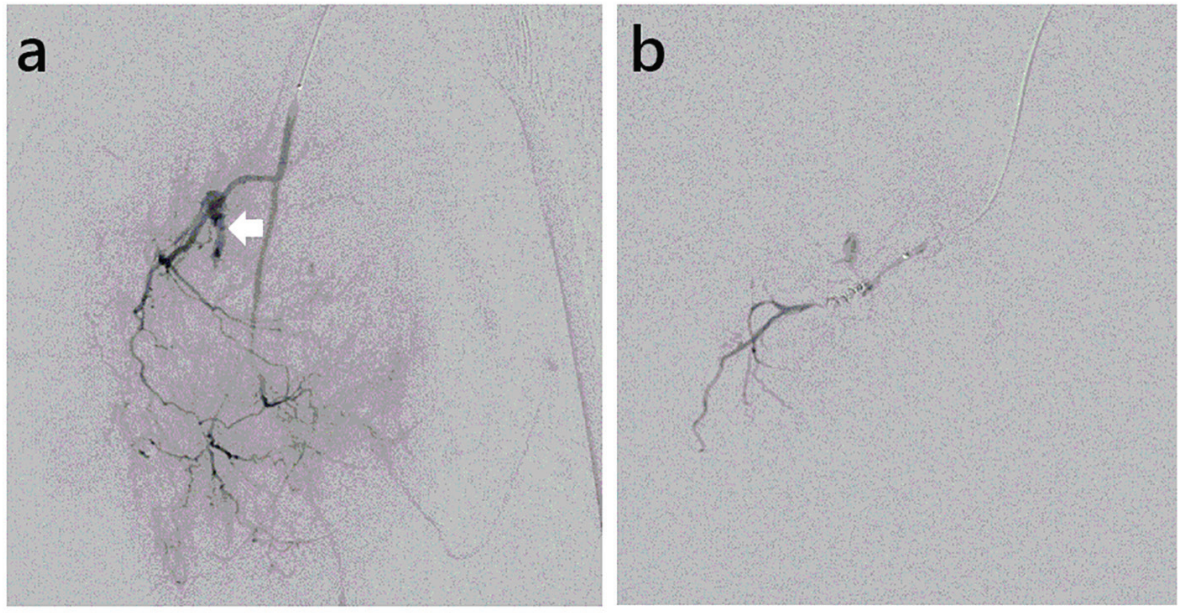

Figure 3. Selective angiogram of the left deep femoral artery, showing the pseudoaneurysm. (a) Before coil embolization. Extravasation of contrast medium from pseudoaneurysm (arrow). (b) After coil embolization.

it is difficult to accurately diagnose rupture of a pseudoaneurysm with hematoma [11]. Furthermore, it is difficult to obtain information on muscle damage after rupture of a pseudoaneurysm on color Doppler imaging. Several case reports have documented that pseudoaneurysm of the femoral artery can lead to compartment syndrome [12-14]. In the absence of appropriate treatment, compartment syndrome can in turn cause permanent muscle and nerve damage. Although our patient had a swollen left adductor muscle caused by hematoma, early diagnosis of the large hematoma in the adductor muscle was possible on contrast-enhanced $\mathrm{CT}$, and angiography confirmed the presence of a pseudoaneurysm, allowing early treatment to be performed, thereby preventing the development of compartment syndrome.

In conclusion, our experience indicates that rupture of a pseudoaneurysm of the deep femoral artery can occur in patients who are receiving warfarin, even when the INR value is maintained within the therapeutic range. Swelling of the thigh caused by rupture of a deep femoral pseudoaneurysm resembles the lower-extremity edema occurring after pelvic lymph-node dissection. We found that contrast-enhanced CT was useful for the diagnosis of pseudoaneurysm and for evaluation of the extent of muscle damage. Some cancer patients have venous thrombosis and require anticoagulation therapy. In cancer patients with risk factors for pseudoaneurysm who are receiving warfarin, physicians should consider the risk of pseudoaneurysm formation, even when the dose of warfarin is maintained within the therapeutic INR range.

\section{Financial Support}

None.

\section{Conflicts of Interest}

None.

\section{References}

1. Dillon JP, O'Brien GC, Laing AJ, Adelowokan T, Dolan M. Pseudoaneurysm of the profunda femoris artery following an inter-trochanteric fracture of the femur. Injury Extra. 2004;35:30-32.

2. Naouli H, Jiber H, Bouarhroum A. False aneurysm of perforating branch of the deep femoral artery-Report of two cases. Int J Surg Case Rep. 2015;14:36-39.

3. Lee YB, Cho YH, Lee JH, Lee JH, Kim MS, Kim JH, Lim $\mathrm{CW}$, et al. Endovascular Management of Spontaneous Superficial Femoral Artery Pseudoaneurysm in a Renal Allograft Patient. Lipid Atheroscler. 2014;3(1):49-53.

4. Azghari A, Bouayad M, Belmir H, Tijani Y, Bouziane Z, Idrissi R, El Mahi O, et al. [Vascular type of EhlersDanlos syndrome: a rare cause of spontaneous pseudoaneurysms]. J Mal Vasc. 2010;35(4):254-258.

5. Kuruvilla M, Gurk-Turner C. A review of warfarin dosing and monitoring. Proc (Bayl Univ Med Cent). 2001;14(3):305-306.

6. Dubinsky IL. Hematoma of the rectus abdominis muscle: case report and review of the literature. J Emerg Med. 1997;15(2):165-167.

7. Ma M, Snook CP. Ruptured femoral pseudoaneurysm presenting as a lateral abdominal wall hematoma. J Emerg Med. 2005;29(2):147-150.

8. Behera C, Naagar S, Krishna K, Taraporewalla DR, Garudadhri GV, Prasad K. Sudden death due to ruptured pseudoaneurysm of femoral artery in injected drug abusers report of four cases at autopsy and review of literature. $\mathrm{J}$ Forensic Leg Med. 2014;22:107-111.

9. Goh BK, Chen CY, Hoe MN. Bilateral spontaneous rupture of the muscular branch of the superficial femoral artery with pseudoaneurysm formation. Ann Vasc Surg. 2004;18(6):736-739.

10. Coughlin BF, Paushter DM. Peripheral pseudoaneurysms: evaluation with duplex US. Radiology. 1988;168(2):339342 . 
11. Demirbas O, Batyraliev T, Eksi Z, Pershukov I. Femoral pseudoaneurysm due to diagnostic or interventional angiographic procedures. Angiology. 2005;56(5):553-556.

12. Young PS, Middleton RG, Shaw AD. Profunda femoris artery pseudoaneurysm complicating compartment syndrome after blunt trauma. Am J Sports Med. 2011;39(8):1786-1788.
13. Karkos CD, Hughes R, Prasad V, D'Souza SP. Thigh compartment syndrome as a result of a false aneurysm of the profunda femoris artery complicating fixation of an intertrochanteric fracture. J Trauma. 1999;47(2):393-395.

14. Langen RP, Ruggieri R. Acute compartment syndrome in the thigh complicated by a pseudoaneurysm. A case report. J Bone Joint Surg Am. 1989;71(5):762-763. 